\title{
DNA REPAIR, GENETIC INSTABILITY, AND CANCER
}


This page intentionally left blank

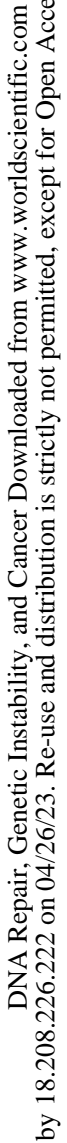



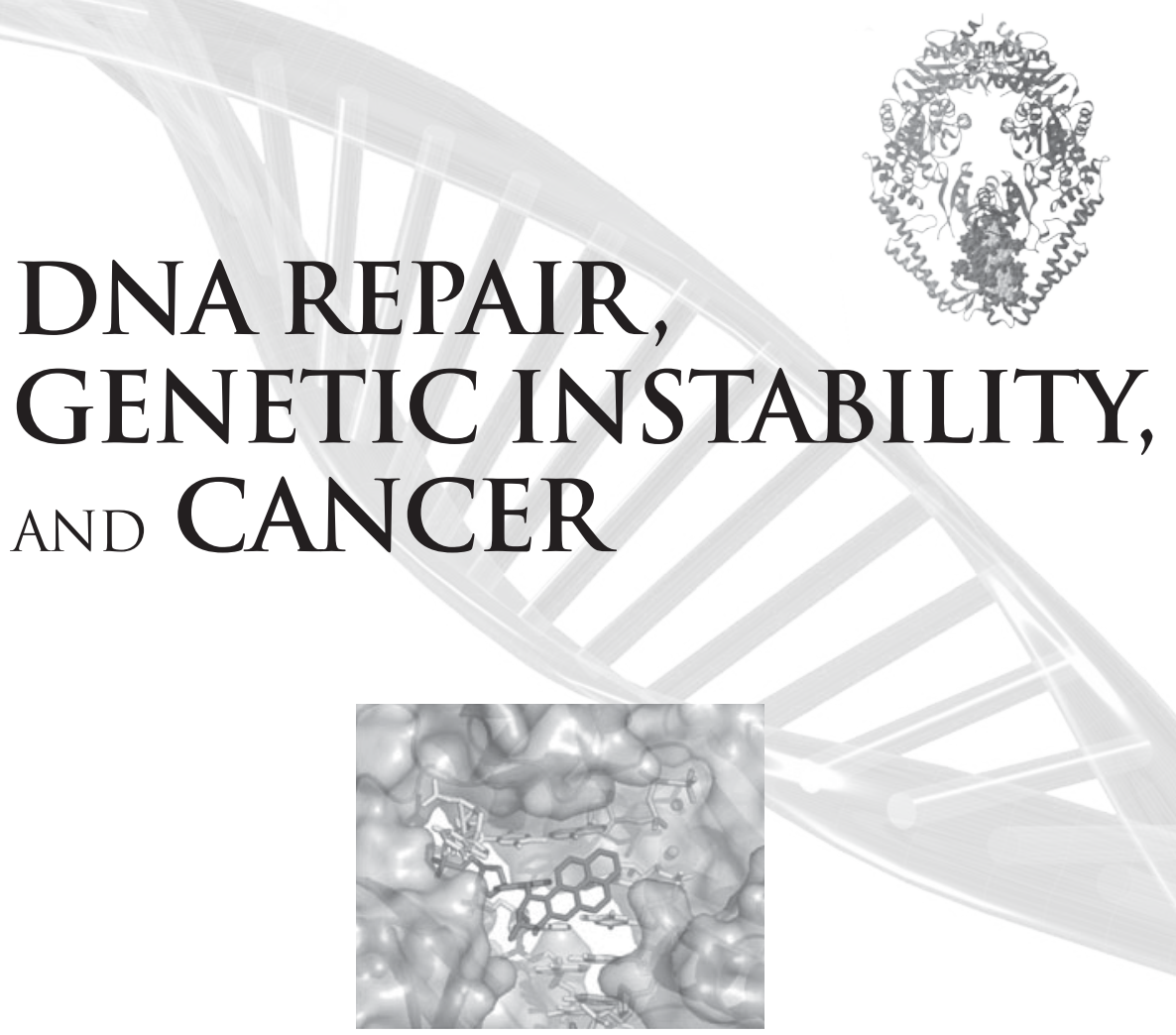

EDITORS

\title{
QINGYI WEI
}

\author{
LEI LI
}

The University of Texas M.D. Anderson Cancer Center, Houston, USA

\section{DAVID J CHEN}

The University of Texas Southwestern Medical Center, Dallas, USA

\section{No World Scientific}




\title{
Published by
}

World Scientific Publishing Co. Pte. Ltd.

5 Toh Tuck Link, Singapore 596224

USA office: 27 Warren Street, Suite 401-402, Hackensack, NJ 07601

UK office: 57 Shelton Street, Covent Garden, London WC2H 9HE

\author{
Library of Congress Cataloging-in-Publication Data \\ DNA repair, genetic instability, and cancer / [edited by] Qingyi Wei, \\ Lei Li, David J. Chen. \\ p. cm. \\ Includes bibliographical references and index. \\ ISBN-13 978-981-270-014-8 -- ISBN-10 981-270-014-5 \\ 1. Neoplasms--etiology. 2. Neoplasms--genetics. 3. DNA Damage--genetics. \\ 4. DNA Repair--genetics. 5. DNA Repair-Deficiency Disorders. \\ 6. Genetic Predisposition to Disease. I. Wei, Qingyi. II. Li, Lei. III. Chen, David. \\ IV. Title. \\ QH467.D164 2006 \\ 616.99'4042--dc22 \\ 2006050159
}

\section{British Library Cataloguing-in-Publication Data}

A catalogue record for this book is available from the British Library.

Copyright (C) 2007 by World Scientific Publishing Co. Pte. Ltd.

All rights reserved. This book, or parts thereof, may not be reproduced in any form or by any means, electronic or mechanical, including photocopying, recording or any information storage and retrieval system now known or to be invented, without written permission from the Publisher.

For photocopying of material in this volume, please pay a copying fee through the Copyright Clearance Center, Inc., 222 Rosewood Drive, Danvers, MA 01923, USA. In this case permission to photocopy is not required from the publisher.

Typeset by Stallion Press

Email: enquiries@ stallionpress.com

Printed in Singapore. 


$$
\begin{aligned}
& \text { In memory of Dr. Lawrence (Larry) Grossman, } \\
& \text { one of the great pioneers and leaders in the field of DNA repair, } \\
& \text { who passed away on January 13, } 2006
\end{aligned}
$$


This page intentionally left blank 


\section{Preface}

Human DNA is constantly bombarded by endogenous (e.g. reactive oxygen species) and exogenous (UV, ionizing radiation and reactive chemicals) carcinogens. To ensure an accurate passage of genetic information onto daughter cells, cells have evolved elaborate surveillance systems and various DNA repair mechanisms that respond to the harmful stimuli and prevent damaged DNA from being converted to heritable mutations. Over the past 30 years, major frameworks have been established for major DNA repair pathways, including base-excision repair (BER), nucleotide excision repair (NER), mismatch repair (MMR), homologous recombination (HR), and non-homologous end-joining (NHEJ).

Since apurinic/apyrimidinic (AP) endonuclease and uracilDNA glycosylase (UDG), the two enzymes involved in BER were discovered in Escherichia coli in the early 1970s, over 20 proteins have been identified as the core and accessory proteins of BER that primarily targets alkylated, deaminated, and oxidized bases, with a certain degree of substrate overlap with other pathways. In contrast, the NER pathway is much more versatile, and is a predominant mechanism protecting cells from UV- and chemical-induced bulky DNA lesions that are often mutagenic. To date, more than 30 genes have been identified that participate in NER. MMR, on the other hand, is an important genome caretaker system. It ensures genomic stability by correcting mismatches generated during DNA replication and 
recombination, suppressing homologous recombination, and triggering apoptosis of cells with severe DNA damage. In response to DNA double-strand breaks, the most dangerous lesions, HR may be used to repair the damage. HR plays critical roles in mitotic cells in repairing DNA double-strand breaks and interstrand crosslinks and in restarting replication forks blocked by DNA lesions produced by both reactive intermediates of normal cellular metabolism, exogenous chemicals, and radiation. However, NHEJ is the predominant repair pathway for removing DNA double-strand breaks in mammalian cells. To survive from lesions that block DNA replication, cells have also evolved a pathway that allows for damage tolerance or lesion bypass with high or low fidelity. A key question is how the cell cycle checkpoint machinery detects and signals the presence of damaged DNA that is embedded in millions to billions of normal base pairs. Partial answers come from recent structural and functional studies that reveal atomic details of DNA repair protein and nucleic acid interactions.

The hallmark of cancer is genomic instability that may be initiated from DNA damage and faulty DNA repair systems. The pathogenesis of cancer, which is frequently an environmentally induced disease, reflects the outcome of disrupted balances among diverse biological systems, including those that govern cell growth and proliferation, signal transduction, DNA damage and repair, cell cycle checkpoint and control, and apoptosis in response to environmental insults. Yet, each individual is genetically unique and his or her responses to environmental risk factors or hazards are also unique.

The role of DNA repair in the etiology of cancer has been well illustrated in several hereditary syndromes, in which an inherited defect in DNA repair and related biological processes is associated with extraordinarily high incidence of cancer. For example, patients with xeroderma pigmentosum (XP) have germline mutations in NER genes and have more than 100-fold increased risk of UV-induced skin cancers; patients with hereditary non-polyposis colon cancer (HNPCC) have a defect in MMR due to germline mutations; and patients with Fanconi anemia (FA) appear to be sensitive 
to agents that cause DNA-crosslinks and have 500-fold increased risk of developing squamous cell carcinomas of the head and neck.

However, associations between inherited DNA repair defect and risk of cancer have not always been apparent in the general population. In the past 10 years, there has been a growing body of literature that begins to address this important research question at the population level. More recently, the discovery of single nucleotide polymorphisms (SNPs) in DNA repair genes has inspired a wave of association studies, some of which established a genetic basis for a suboptimal repair phenotype in the general population. These findings provide a rationale that by genetic screening for functional SNPs, it may be feasible to identify at-risk populations who can be targeted for primary prevention of cancer that has an etiology of genetically determined variation in DNA repair.

To achieve the goal of eradicating cancer, it is paramount to understand the underlying molecular mechanisms for the maintenance of genetic stability. This book provides a snapshot of our current understanding of DNA damage repair and recent advances in the research of DNA repair, genetic instability and cancer.

Qingyi Wei

Lei Li

David Chen 
This page intentionally left blank 


\section{Contributors}

Sandeep Burma, PhD

Assistant Professor

Division of Molecular Radiation Biology

Department of Radiation Oncology

University of Texas Southwestern Medical Center 5801 Forest Park Road

Dallas, TX 75390-9187

Sandeep.Burma@UTSouthwestern.edu

Benjamin Chen, PhD

Assistant Professor

Division of Molecular Radiation Biology

Department of Radiation Oncology

University of Texas Southwestern

Medical Center

5801 Forest Park Road

Dallas, TX 75390-9187

Benjamin.Chen@utsouthwestern.edu 
David J. Chen, PhD

Professor and Director

Division of Molecular Radiation Biology

Department of Radiation Oncology

University of Texas Southwestern

Medical Center

5801 Forest Park Road

Dallas, TX 75390-9187

david.chen@utsouthwestern.edu

Junjie Chen, PhD

Professor

Department of Therapeutic Radiology

Hunter Bldg. Room 213C

Yale University School of Medicine

333 Cedar Street, P.O. Box 208040

New Haven CT 06520-8040

Junjie.Chen@yale.edu

Rong Guo, PhD

Post-Doctoral Fellow

Lab of Genetics

Genome Stability and Chromatin Remodeling Section

National Institute on Aging/NIH

333 Cassell Drive, TRIAD Building RM. 3000

Baltimore, MD 21224

Tel.: 410-558-8489

Fax : 410-558-8331

gor@grc.nia.nih.gov 
Bo Hang, MD, PhD

Staff Scientist

Department of Molecular Biology

Life Sciences Division

Lawrence Berkeley National Laboratory

University of California

Berkeley, CA 94720

Bo_hang@lbl.gov

Zhibin $\mathrm{Hu}, \mathrm{MD}, \mathrm{PhD}$

Post-Doctoral Fellow

Department of Epidemiology

The University of Texas M. D. Anderson

Cancer Center

1515 Holcombe Blvd.

Houston, Texas 77030

zbhu@mdanderson.org

Maxwell P. Lee, PhD

Investigator

Lab Population Genetics (HNC7Z35)

National Cancer Institute

Building 41, Room D702C

41 Library Drive Room D702

Bethesda, MD 20892-5060

ml110b@nih.gov

Guo-Min Li, PhD

Professor

Departments of Toxicology and Pathology

University of Kentucky Medical Center

125 Health Sciences Research Building

800 Rose Street

Lexington, KY 40536

gmli@uky.edu 
Lei Li, PhD

Associate Professor

Department of Experimental Radiation

Oncology

The University of Texas M. D. Anderson

Cancer Center

1515 Holcombe Blvd.

Houston, Texas 77030

leili@mdanderson.org

Jac A. Nickoloff, PhD

Professor and Chairman

Department of Molecular Genetics and

Microbiology

CRF 127

University of New Mexico HSC

915 Camino de Salud NE

Albuquerque, NM 87131

jnickoloff@salud.unm.edu

Binghui Shen, $\mathrm{PhD}$

Professor and Director

Department of Radiation Biology

City of Hope National Medical Center and

Beckman Research Institute

1500 East Duarte Road

Duarte, CA 91010

bshen@coh.org 
Zhiyuan Shen, PhD

Associate Professor and Chief

Division of Radiation Cancer Biology

Department of Radiation Oncology

Robert Wood Johnson Medical School

Cancer Institute of New Jersey

University of Medicine and Dentistry of New Jersey

195 Little Albany St

New Brunswick, NJ 08903-2681

shenzh@umdnj.edu

Purnima Singe, PhD

Research Fellow

Department of Radiation Biology

City of Hope National Medical Center and

Beckman Research Institute

1500 East Duarte Road

Duarte, CA 91010

PSingh@coh.org

Li-E Wang, MD

Instructor

Department of Epidemiology

The University of Texas M. D. Anderson

Cancer Center

1515 Holcombe Blvd.

Houston, Texas 77030

lwang@mdanderson.org 
Weidong Wang, PhD

Senior Investigator

Genome Instability and Chromatin Remodeling Section

National Institute on Aging/NIH

333 Cassell Drive, TRIAD Building RM. 3000

Baltimore, MD 21224

wangw@grc.nia.nih.gov

Qingyi Wei, MD, PhD

Professor

Department of Epidemiology - 1365

The University of Texas M. D. Anderson Cancer Center 1515 Holcombe Blvd.

Houston, Texas 77030

qwei@mdanderson.org

Jamie Wood

Department of Therapeutic Radiology

Hunter Bldg. Room 210

Yale University School of Medicine

333 Cedar Street, P.O. Box 208040

New Haven CT 06520-8040

Jamie.Wood@yale.edu 
Wei Xiao, PhD

Professor and Head

Department of Microbiology and Immunology

College of Medicine

University of Saskatchewan

107 Wiggins Road

Saskatoon, SK, S7N 5E5 Canada

wei.xiao@usask.ca

Fang Xu

Professor

Department of Biology

Ningxia Medical College

692 Sheng-Li Road

Yinchun, Ningxia 750004, China

xufang513@yahoo.com.cn

Wei Yang, PhD

Senior Investigator

Laboratory of Molecular Biology

Molecular Structure Section (HNK6C5)

NIDDKD/NIH

Building 5, Room B107

5 Memorial Dr.

Bethesda, MD 20892

Wei.Yang@nih.gov 
Yingnian Yu, MD

Professor

Department of Pathophysiology

Center of Environmental Genomics

Zhejiang University School of Medicine

353 Yan'an Road

Hangzhou, Zhejiang 310031, China 


\section{Contents}

Preface vii

Contributors $x i$

Chapter 1 DNA Damage Sensing and Signaling 1

Jamie L. Wood and Junjie Chen

Chapter 2 Base Excision Repair

Bo Hang

Chapter 3 Nucleotide Excision Repair

Lei Li

Chapter 4 DNA Mismatch Repair: Biological Functions and Molecular Mechanisms

Guo-Min Li

Chapter 5 Mammalian Homologous Recombination Repair and Cancer Intervention

Zhiyuan Shen and Jac A. Nickoloff

Chapter 6 Role of Non-Homologous End Joining in the Repair of DNA Double-Strand Breaks 
Chapter 7 A DNA-damage Response Network of Fanconi Anemia and BRCA Proteins

Rong Guo and Weidong Wang

Chapter 8 Structure and Function of DNA Repair Proteins: Lesion Recognition

Wei Yang

Chapter 9 DNA Damage Tolerance and Translesion

Synthesis

Fang Xu, Yingnian Yu and Wei Xiao

Chapter 10 Nucleases in DNA Repair, Replication and Recombination: Flap Endonuclease-1 as a Paradigm

Purnima Singh and Binghui Shen

Chapter 11 Integrative Genomics and Epigenomics: Application in Cancer Research Maxwell P. Lee

Chapter 12 Molecular Epidemiology of DNA Repair and Cancer Susceptibility - A Review of Population-Based Studies Zhibin Hu, Li-E Wang and Qingyi Wei

Index 